gesetzt, und die Ergebnisse in unregelmäßig erscheinenden Mitteilungen niedergelegt, über welche fortan in dieser Zeitschrift referiert werden soll.

\section{Die Novelle zur Gewerbeordnung und der Entwurf eines Gesetzes über Arbeitskammern.}

\author{
Von Dr. O. BonHOEFEER. \\ (Eingeg. d. 21./4. 1908.)
}

Das verflossene Vierteljahr hat der deutschen Industrie zwei Gesetzentwürfe gebracht: Die Novelle zur Gewerbeordnung, am 16./12. 1907 von den verbündeten Regierungen dem Reichstag vorgelegt, und den Entwurf eines Gesetzes über Arbeitskammern, veröffentlicht im Reichsanzeiger vom 4./2. 1908. Beide Entwürfe bringen erhebliche neue Gesichtspunkte für die Industrie und haben infolgedessen je nach dem partei- und wirtschaftspolitischen Standpunkt des Betrachters die verschiedenste Beurteilung gefunden.

I. Die Novelle zur Gewerbeordnung enthält, wie in der Natur der Sache liegt, ein Konglomerat von Bestimmungen von verschieden starkem Interesse für die verschiedenen Industrien. Sie befaßt sich v. a. mit der Zeugniserteilung, mit den Lohnbüchern, Fortbildungsschulzwang, Regelung der Arbeitszeit und Pausen in gesundheitsschädlichen Betrieben, mit der Konkurrenzklausel, der Anrechnung der Krankengelder auf den Gehalt während der Erkrankung, regelt neu die Arbeitszeit der Arbeiterinnen und bringt schließlich ein Gesetz über Hausindustrie.

Nach $\S 113$, Abs. 1, kann die Erteilung eines $Z$ eugnisses künftighin schon vom Tage der Kündigung $a b$ und nicht erst bei der Entlassung vom Arbeiter verlangt werden, eine Bestimmung, die von keiner Seite nennenswert zu beanstanden ist, wenn, entsprechend einer Eingabe des Vereins zur Wahrung der Interessen der chemischen Industrie, dieses Zeugnis auf Verlangen e rs t be i m A b g $\mathbf{n}$ g auf Führung und Leistung ausgedehnt werden muß.

In das Gebiet des Arbeiterschutzes gehört ebenfalls die von der Novelle vorgeschlagene Anderung der sog. $\mathrm{L}$ o h $\mathrm{n} \mathrm{b}$ ü $\mathrm{ch}$ e $\mathrm{r}$, die bisher nur für die Betriebe der Kleider- und Wäschekonfektion vorgeschrieben sind, und die nach der Novelle nunmehr den Charakter von Abrechnungsbüchern erhalten sollen, indem sie außer den bis jetzt verlangten Eintragungen auch Vermerke über den Zeitpunkt der U̇bertragung und Ablieferung der Arbeit, den zur Auszahlung gelangenden Lohnbetrag und den Tag der Lohnzahlung enthalten müssen. Es wird hierdurch die wünschenswerte Klarheit in den Arbeitsbedingungen der einzelnen Betriebe gesichert.

Von umfassenderer Bedeutung ist die Änderung des $\$ 120, \mathrm{Abs} .3$, dahin, daß künftighin durch die Kommunen die Verpflichtung z u m Besuch einer Fortbildungsachule auch auf Arbeiterinnen unter $18 \mathrm{Jah}$. r e n ausgedehnt werden kann. Die Bedenken, die hiergegen von seiten der solche Arbeiterinnen beschäftigenden Industrie vorgebracht werden, und die sich auf die dadurch hervorgerufene Verkürzung der Arbeitszeit und auf Verminderung der Arbeitsgelegenheit infolge Nichteinstellung solcher Arbeiterinnen beziehen, können nicht in Betracht kommen gegenüber den Vorteilen, die in der verbesserten Ausbildung dieser jugendlichen Arbeiterinnen für die Gesamtheit liegen. Es wird woh] kaum zu bestreiten sein, daB, wenn die weitere Ausbildung in dem durch $\$ 120$, Abs. 2, der G.-O. vorgesehenen Sinne - als Fortbildungsschulen im Sinne dieser Bestimmung gelten auch Anstalten, in welchen Unterricht in weiblichen Hand- und Hausarbeiten erteilt wird - geschieht, dies aus wirtschaftlichen Gründen sehr zu begrüßen ist.

Die Anderung des $\$ 120$ e ist für die chemische Industrie von speziellem Interesse, sie fügt der bisherigen Bestimmung, welchen Anforderungen bestimmte Anlagen in bezug auf Vorrichtungen zum Schutze von Leben und Gesundheit der Arbeiter zu genügen haben, den Zusatz an, daß künftighin in diese Vorschriften auch B e stimm ungen überdas Verhaltender Arbeiterim $\mathrm{B}$ etri e b aufgenommen werden können. Damit ist gesagt, daß küntighin die Beachtung and Ausführung der zum Schutze der Arbeiter erlassenen Vorschriften bei diesen selbst erzwungen werden kann durch Verhängung von Geldstrafen oder Haft nach § 1471 Abs. 4 der G. O., während bisher der Arbeitgeber der Durchführung der von ihm erlassenen Schutz- und Sicherheitsmaßregeln ziemlich machtlos gegenüberstand. Zum Erlaß dieser Vorschriften sind sogar die zuständigen Polizeibehörden eventuell befugt.

Nach $\$ 120 \mathrm{f}$ der G. O. war bisher nur der Bundesrat zu genereller $R$ e ge l ung der A r beitszeit und der Pa $\mathrm{p}$ sen in den Betrieben, in welchen durch übermäBige Dauer der Arbeitszeit die Gesundheit gefährdet wurde, befugt, nunmehr überträgt die Novelle diese Befugnis - soweit bisher nicht schon solche Vorschriften vom Bundesrat erlassen sind - den zuständigen Polizeibehörden. Damit ist letzteren eine Machtbefugnis gegeben, die weit über das bisherige Maß hinausgeht und nach Ansicht eines großen Teils der Arbeitgeberschaft nicht genügend kompensiert wird durch den im letzten Satz des betr. Paragraphen zugestandenen Beschwerdeweg bei der höheren Verwaltungsbehörde. Eine gewissenhafte und sechverständige Prüfung durch die unteren Verwaltungs- und Polizeibehörden vorausgesetzt, kann man sich trotzdem dem Bedenken nicht verschlieBen, daß durch diese Befugnis in den verschiedenen Teilen des Reiches für das gleiche Gewerbe ganz verschiedene Arbeits- und Existenzbedingungen geschaffen werden. Begründet wird diese Änderung von der Regierung damit, daß entgegen dem bisherigen umständlichen und zeitraubenden Verfahren durch BundesratsbeschluB nunmehr die manchmal in kürzester Zeit nötige Ánderung gesichert sei.

Für die chemische Industrie von größter $\mathrm{Be}$ deutung ist die in der Novelle in dem $\$ 133 \mathrm{f}, \mathrm{g}$ und $h$ getroffene Neuregelung der Frage der $\mathbf{K}$ o $\mathbf{n}$ k u rrenzk la u sel. Die Kritik, die in den letzten Jahren gegen die teilweise vorhandene mis- 
bräuchliche Ausnutzung der Konkurrenzklausel einsetzte, wie sie der $\S 133 \mathrm{f}$ der G. O. zuließ, hat das Gute gehabt, daß diese Frage von den beteiligten Kreisen mit aller Gründlichkeit behandelt wurde, und man kann mit Recht die Neufassung dieses Paragraphen als das Produkt dieser langjährigen Erörterungen betrachten. Der $\S 133 \mathrm{f}$ der G. O. ließ bisher eine Vereinbarung ïber Beschränkung der gewerblichen Tätigkeit des Angestellten nach Auflösung des Dienstverhältnisses insoweit zu, als diese Beschränkung nach Ort, Zeit und Gegenstand nicht die Grenzen überschreitet, durch welche eine unbillige Erschwerung des Fortkommens des Angestellten ausgeschlossen wird. Diese allgemeine Fassung ist durch die Novelle nach verschiedenen Richtungen eingeschränkt worden: Die Beschränkung kann auf mehr als drei Jahre von der Beendigung des Dienstverhältnisses an nur dann vereinbart werden, wenn während der Dauer der Beschränkung dem Angestellten das zuletzt von ihm bezogene Gehalt weiter bezahlt wird; eine Karenzzeit kann nicht verlangt werden, wenn der Unternehmer durch vertragswidriges Verhalten dem Angestellten Grund gibt, das Dienstverhältnis gemäß den Vorschriften des $\S 133 \mathrm{~b}$ und $\mathrm{d}$ aufzulösen, $d$. h., wenn wichtige, nach den Umständen des Falles die Aufhebung des Dienstverhältnisses rechtfertigende Gründe fehlen, oder wenn Ehrverletzungen, Nichtgewährung vertragsmäßiger Leistungen oder Leben und Gesundheit gefährdende, bei Eingehung des Dienstverhältnisses nicht erkennbare Umstände eintreten. Die Konventionalstrafe bleibt bestehən, darüber hinaus jedosh Schadenersatz zu verlangen, ist ausgeschlossen. Löst der Unternehmer das Dienstverhältnis auf, ohne daß ein erheblicher, von ihm nicht verschuldeter Anla B vorliegt, so ist die von dem Angestellten eingegangene Konkurrenzklausel nicht verbindlich, es sei denn, daß während der Karenzzeit dem Angesteliten das zuletzt bezogene Gehalt weiter bezahlt wird. Begrenzt ist die Geltung dieser neuen Bestimmungen dadurch, daß sie nur für diejenigen Angastellten gelten, deren Gehalt $8000 \mathrm{M}$ nicht übersteigt, mit Ausnahme der obengenannten Bestimmungen des $\S 133 \mathrm{~b}$ und $\mathrm{d}$, der auch für diese Angestellten in Geltung bleibt.

Wer die Frage der Konkurrenzklausel in der letzten Zeit verfolgt hat, wird den Eindruck gewinnen, daß die Novelle zwischen den vielerlei Wünschen der beiden in Betracht kommenden Faktoren den Mittelweg zu nehmen versucht hat. Von einom Teil der Angestellten, namentlich der Handlungsgehilfen, ist dringend die Aufhebung der Klausel überhaupt verlangt worden mit der Begründung, daß es einerseits unbillig sei, dem Rinzelnen, in diesom Falle dem wirtschaftlich Schwächeren, sein meist einziges Kapital, die erworbenen Kenntnisse und seine Arbeitskraft, lahm zu legen, andererseits, daß es überhaupt unwirtschaftlich sei, einem Betrieb für eine Reihe von Jahren durch die Konkurrenzklausel gewissermaßen eine Monopolstellung zu sichern, statt in freier Entfaltung die Kräfte spielen zu lassen. Demgegenüber wird nahezu ausnahmslos von seiten der Arbeitgeber das völlige Fallenlassen der Konkurrenzklausel als unannehmbar bezeichnet aus Gründen, denen namentlich in Bezug auf die Verhältnisse in der chemischen
Industrie billigerweise nicht wird widersprochen werden können. Die chemische Industrie lebt und gedeiht zum größten Teil durch Erfindungen, die meist unter sehr großen von den Unternehmern gebrachten Opfern an Zeit und Geld durch die Angestellten ausgearbeitet sind, Erfindungen, die zum Teil patentrechtlich nicht geschützt werden können, für die oft aber auch Patentschutz aus Gründen der Geheimhaltung nicht nachgesucht werden kann, und die also als Fabrikgeheimnis bewahrt werden mïssen. Es ist nun keinesfalls zu verlangen, daß dem Unternehmer diese Werte ungeschützt bleiben sollen. Dieser Standpunkt ist auch in der Novelle eingenommen und die Vereinbarung einer Konkurrenzklausel beibehalten worden.

Bedauerlich dagegen bleibt für die Allgemeinheit der Angestellten, daß der auf der letzten Hauptversammlung unseres Vereins eingenommene Standpunkt keinen Ausdruck in der Novelle gefunden hat, daß die Gehaltszahlung während der Karenzzeit fortzusetzen ist, und daß unter Umständen mit der Fortzahlung des Gehalts allein noch kein genügendes Äquivalent für die Beschränkung des Angestellten durch die Konkurrenzklausel geschaffen ist, sondern daß es nicht mehr als billig ist, daß außer dem Gehalt auch eine Vergütung für die dem Angestellten in Form von Tuntiemen, Gratifikationen usw. zufließenden Bezüge gewährt wird, weil diese im Budget desselben eine große Rolle spielen. Dieser Auffassung sind bekanntlich die Arbeitgeber der chemischen Industric gerecht geworden, indom sie auf ihrer 30. Hauptversammlung in Lübeck am 13./9. 1907 auf Grund cines Referates des Vorsitzenden unseres Vereins, Professor Dr. D u is ber.g - Elberfeld, einstimmig die Resolution angenommen haben, daß dem technischen Angestellten, wenn von $\mathrm{ihm}$ die Einhaltung einer Karenzzeit verlangt wird, das zuletzt bezogene feste Gehalt, mindestens aber eine Entschädigung zugesichert wird, die $\mathrm{ihm}$ eine seiner $\mathrm{St}$ e $1 \mathrm{lu} \mathrm{ng}$ entsprechende Lebensführung ermöglicht. Die chemische Industrie ist also in zwei Hauptpunkten zugunsten ihrer technischen Angestellten über die Novelle hinausgegangen, einmal, daß es ohne Vergütung tiberhaupt keine Karenzzeit geben soll, und dann, daß diese Vergütung so bemessen sein muß, daß dem unter Karenz stehenden Angestellten ein Leben seiner bisherigen Stellung angemessen ermöglicht wird.

Für den Gültigkeitsbereich der Schutzbestimmungen der Gewerbeordnung ist von Bedeutung, daß die Novelle in $\S 134$ den Begriff ,F a b r i k6", der auf dem Grenzgebiet zwischen Industrie und Handwerk fortgesetzt zweifelhaft war und in der Rechtsprechung infolgedessen zu den verschiedensten Urteilen geführt hat, fallen läßt und dafür die Einteilung nach Betrieben mit $\mathbf{m i n d e}$. ste $\mathbf{n}$ 10, b z w. 20 A r b e it e r n einführt.

Neben weiteren Bestimmungen, die eine Entlassung eines Angestellten erst im Falle einer acht Wochen - gegen früher sechs Wochen - übersteigenden militärischen Dienstleistung zulassen, die die Anrechnung der während einer Erkrankung erhaltenen $\mathrm{B}$ e $\mathrm{z} \ddot{\mathrm{u}} \mathrm{g}$ e a $\mathrm{u}$ s e in e r g e setzlich vorgesehriebenen Krankenoder Unfallversicherung erlauben und die Geha $1 \mathrm{tsz}$ ah $1 \mathrm{un}$ ge n normaler Weise auf 
den Monatsschluß festlegen, keinenfalls aber in länger als vierteljährigen Zeitabschnitten zulassen, sind von größerer Bedeutung das Verbot d e r $\mathrm{Nachtarbeit}$ and die Festsetzung einer Maximalarbeitszeit für Arbeite. r i n n e n von 10 Stunden täglich, vom 1./1. 1910 ab unter Zulassung besonderer Änderungen durch den Reichskanzler, die jedoch auch durch eine zulässige Gesamtzahl von 60 Stunden pro Woche und 11 Stunden im Maximum pro Tag begrenzt sind. Diese Bestimmung berührt hauptsächlich die Textilindustrie und wird auch von einem Teil der Unternehmer dieses Gewerbes bekämpft, weil sie, über die Vorschläge der Berner Konvention hinausgehend, die einheimische Industrie gegenüber der des Auslandes mehr belastet. An einer Annahme dieser Bestimmung durch den Reichstag ist im übrigen nicht $\mathrm{zu}$ zweifeln, um so weniger, als eine Aufstellung der preuBischen Gewerbeinspektionen nachweist, daß bereits jetzt in $71 \%$ aller Betriebe in Preußen ca. $62 \%$ aller Arbeiterinnen den Zehnstundentag oder noch kürzere Arbeitszeit haben.

In den $\$ 139 \mathrm{n}$ bis $139 \mathrm{y}$ betritt die Novelle ein bis jetzt gesetzlich nicht gefaßtes Gebiet, das der $\mathrm{H}$ a u s ind ustri e. Seit einer langen Reihe von Jahren wird in der sozialpolitischen Presse und Literatur immer wieder darauf hingewiesen, unter welch unglüoklichen Verhältnissen in ethischer und wirtschaftlicher Beziehung der größte Teil der Heimarbeiter sein Leben fristet, und es ist das unbestrittene Verdienst der im Jahre 1906 in Berlin in Szene gesetzten Ausstellung der Heimindustrie, die Augen der Allgemeinheit auf diesen wundesten Punkt unseres Wirtschaftslebens gelenkt zu haben. Das Interesse, das diese Ausstellung wohl mit durch den Besuch Ihrer Majestät der Kaiserin in den Kreisen der Regierung gefunden hat, hat vermutlich dazu beigetragen, den Versuch einer gesetzlichen Regelung der Hausindustrie zu beschleunigen. Eine allen Anforderungen gerecht werdende Bearbeitung dieser Materie geboten zu haben, beansprucht die Novelle wohl nicht, es ist ein erster Versuch, der als solcher gewürdigt werden muß. Die Schwierigkeit einer befriedigenden Lösung dieser Frage liegt hauptsächlich darin, daß auf der einen Seite der Hausindustrie der Schutz zuteil werden soll, der den Arbeitern der unter die G. O. fallenden Industrien in rechtlicher und hygienischer Beziehung durch das Gesetz gewährleistet wird, während auf der anderen Seite diesen Absichten gewisse, teilweise sehr enge, Grenzen in lokaler und technischer Beziehung gezogen sind, wenn man nicht ganze Industrien vernichten und Hunderttausende von Existenzen brotlos machen will, Existenzen, die für andere Arbeit nicht mehr tauglich sind, oder denen andere Arbeit nicht geboten werden kann.

So beschränkt sich die Novelle vorerst auf Schutzbestimmungen für die Hausarbeiter in bezug auf Betriebssicherheit usw. der Werkstätten, auf hygienische Vorschriften betr. Luft, Staub und dgl. in den Betrieben, die mit besonderen Gefahren für Leben und Gesundheit verbunden sind, andererseits auf den Schutz der Konsumenten, indem die Hausindustrie für Genuß- und Nahrungsmittel so eingerichtet sein muß, daß Gefahren für die öffentliche Gesundheit ausgeschlossen sind. Läßt sich durch polizeiliche Vorschriften die Gefahr für die Haus. arbeiter selbst oder für die öffentliche Gesundheit nicht vermeiden, so kann durch Bundesratsbeschluß die Verrichtung solcher Hausarbeit ganz verboten werden. Wie weit hier im Interesse der genannten vorgegangen werden muß, wird erst die Erfahrung zeigen. Daran schließen sich weiter für Gewerbetreibende, die außerhalb ihrer Betriebsstätten Hausarbeit verrichten lassen, Bestimmungen über Führung von Listen der beschäftigten Personen, über Beaufsichtigung dieser Werkstätten durch den Arbeitgeber und über Beschränkung der Ausgabe von Hausarbeit auf solche Werkstätten, die gewissen Anforderungen genügen. Ein Beispiel, wie schwierig die Durchführung einzelner dieser Bestimmungen sich gestalten würde, gibt eine Eingabe einer rheinischen Handelskammer, in deren Gebiet die Haus. industrie eine große Rolle spielt; sie führt an, daß eine ihrer Firmen $600-700$ Hausweber in 30 Bürgermeistereien beschäftigt, also bei der als Minimum vorgeschriebenen halbjährlichen Kontrolle 1200 bis 1400 Revisionen auszuführen hätte.

Mit einer Reihe Bestimmungen über Str a f v e r s c h ärf ung en gegen Arbeitgeber, die sich wiederholter Zuwiderhandlungen gegen die Schutzvorschriften schuldig machen, schließt die Novelle.

Der Reichstag hat inzwischen die Novelle durchberaten und einer Kommission von 28 Mitgliedern überwiesen, deren Aufgabe es nun sein wird, aus den verschiedenen widerstreitenden Ansichten und Auffassungen das für die Gesamtheit und die einzelnen Industrien zweckmäBigste dem Plenum zur Annahme rorzuschlagen.

II. Am 4./2. dieses Jahres wurde im Reichs. anzeiger der Entwurf eines Gesetzes über Arbeitskammern veröffentlicht, und damit eine Materie der öffentlichen Kritik unterbreitet, die sich seit einer langen Reihe von Jahren in Parlament, Presse und sozialpolitischen Vereinen der eingehendsten Behandlung erfreute. 18 Jahre früher am 4./2. 1890 - hatte ein kaiserlicher Erlaß die Schaffung von Organen in Aussicht gestellt, ,in denen die Arbeiter durch Vertreter, die ihr Vertrauen besitzen, zur Wahrnehmung ihrer Interessen bei Verhandlungen mit den Arbeitgebern in gemeinsamen Angelegenheiten, sowie ebenso mit den Organen der Regierung befähigt werden."

Das Produkt dieser langjährigen Erörterungen stellt nun der vorliegende Entwurf vor, und wenn der Staatssekretär des Innern, Dr. v. B e t h m a n n$\mathrm{H}$ o $1 \mathrm{lweg}$, trotz des in Unmenge vorliegenden Materials an Vorschlägen zur Einrichtung solcher Organe den bis dahin nicht üblichen Weg beschritten hat, gleichzeitig mit der Weitergabe an den Bundesrat den Entwurf zu veröffentlichen und damit zur Kritik der beteiligten Kreise zu stellen, so mag schon das als ein Beweis angesehen werden, wie schwierig eine befriedigende Lösung der Frage der Regierung erscheint, und wie sie Wert darauf legt, nochmals auf Grund eines Entwurfs das Für und Wider zu hören.

Und in der Tat, wenn die Veröffentlichung nicht den genannten Zweck gehabt hätte, müßte die Regierung heute schon den Entwurf ad acta legen, denn viel ist nicht übrig geblieben, was den beteiligten Kreisen an dem Entwurf annehmbar erscheint. 
Die nachfolgenden Ausführungen sollen den Lesern dieser Zeitschrift in kurzen Zügen die Grundlinien des Entwurfs und die Grundgedanken der an ihm geübten Kritik bieten.

Entsprechend dem Wortlaut des oben angeführten kaiserlichen Frlasses handelt es sich darum, den Arbeitnehmern eine gesetzlich sanktionierte Vertretung zu schaffen, die sie zu Verhandlungen mit den Arbeitgebern und den Behörden bzw. Kommunen in gemeinsamen Angelegenheiten befähigt. Dafür war als erste Frage zu erledigen, wie geschieht das am besten: getrennt jede einzelne der beiden Interessengruppen für sich oder in Organen, die beide Teile in einer Kammer vereinigen? Die Frage ist lange umstritten gewesen und von beiden Teilen zu verschiedenen Zeiten verschieden beantwortet worden. Die Regierung entschied sich für die Vereinigung beider Teile in einer Verhandlungsgemeinschaft und legte den Entwurf einer „Arbeitskammer", nicht einer „Arbeiterkammer" vor. Wenn man die Berechtigung dieser Auffassung prüfen will, muß ins Auge gefaßt werden, was ist der $Z$ we ck dieser A r beitskammern, was sollen sie leisten? Hierüber geben die $\$ 2-6$ des Lntwurfs Aufschluß, in denen als Aufgabe vorangestellt ist: „Die Arbeitskammern sind berufen, den wirtschaftlichen Frieden zu pflegen. Sie sollen die gemeinsamen gewerblichen und wirtschaftlichen Interessen der Arbeitgeber und Arbeitnohmer der in ihnen vertretenen Gewerbezweige, sowie die auf dem gleichen Gebiete liegenden besonderen Interessen der beteiligten Arbeitnehmer wahrnehmen", und weiter : ,Insonderheit gehört zu den Aufgaben der Arbeitskammern ein gedeihliches Verhältnis zwischen Arbeitgeber und Arbeitnehmer zu fördern". Es wird nicht zu leugnen sein, der Gedanke der Regierung ist an sich richtig, daß gemeinsame Interessen zwischen sich gegenüberstehenden Parteien am besten zur Ủbereinstimmung gebracht werden können, wenn beide Teile in direkter Verhandlung, in direkter Rede und Gegenrede einander gegenüberstehen. Voraussetzung bleibt jedoch, daß beide Teile nach Lage der Verhältnisse die Fähigkeit oder die Möglichkeit haben, sich zu einigen!

Der Gedanke dieser gemeinsamen Arbeitskammer schien nahezu allgemein als richtig angenommen zu sein, anch der Reichstag hatte sich gelegentlich in seiner Mehrheit hierfür ausgesprochen; auffallenderweise bekämpfen ihn heute die sozialdemokratische Partei und die Gewerkschaften, nachdem sie jahrelang dafür eingetreten waren; man könnte das vielleicht als ein Zeichen nehmen, da $B$ die Regierung das Riehtige getroffen hat, weil ihr Motiv der Friede zwischen Arbeitgeber und Arbeitnehmer ist, was man jedenfalls von der Sozialdemokratie wohl kaum wird behaupten können. Als Grund der Ablehnung wird von dieser Seite angegeben, daß die Arbeitervertreter in gemeinsamer Kammer aus Furcht vor Maßregelung vielleicht nicht das nötige Rückgrat den Arbeitgebern gegenüber zeigen werden.

Aber auch die Organisationen der Arbeitgeber lehnen zum größten Teil die gemeinsame Arbeitskammer ab, nicht weil der Wille zum Frieden fehlt, sondern weil naeh ihrer Ansicht ein sehr begründeter Zweifel berechtigt ist, ob die Arbeitnehmerbeisitzer sich so unabhängig von parteipolitischen und gewerkschaftlichen Rücksichten werden machen können, daß sie objektiv und sachlich die gestellten Aufgaben erledigen würden. Zu dieser Auffassung führt die Arbeitgebervertretung noch ein weiterer Punkt: Als weitere Aufgaben der einzurichtenden Kammern sind in $\$ 3$ und folgenden vorgesehen: Die Staats- und Gemeindebehörden durch tatsächliche Mitteilungen and Erstattung von Gutachten zu unterstützen, statistische Erhebungen über wirtschaftliche Fragen der von ihnen vertretenen Gewerbezweige vorzunehmen, Gutachten zu erstatten über Auslegung von Verträgen, über Erfüllung von Verbindlichkeiten zwischen Arbeitgebern und Arbeitnehmern, über Verkehrssitten, ferner Vorschläge zu machen über Wohlfahrtseinrichtungen, über Maßnahmen zur Hebung der wirtschaftlichen Lage der Arbeitnehmer und schließlich als Einigungsamt bei Streitigkeiten $\mathrm{zwischen} \mathrm{Arbeitgebern} \mathrm{und} \mathrm{Ar-}$ beitnehmern zu fungieren, wenn es an einem hierfür zuständigen Gewerbegericht fehlt, oder die beteiligten Arbeitnehmer in den Bezirken mehrerer Gewerbegerichte beschäftigt sind, oder wenn die Einigungsverhandlungen bei dem zuständigen Gewerbegericht erfolglos verlaufen sind.

Man sieht, eine Fülle von Aufgaben, die teilweise von schwerwiegender Bedeutung werden, und die in ihrer Entwicklung darauf hinauslaufen können, daß die Arbeitskammern schließlich zu Schiedsämtern sich auswachsen mit allen Imponderabilien, die solch einem Institut in den Augen der Allgemeinheit zukommen. Hierin gerade sehen die die Arbeitskammern ablehnenden Körperschaften der Arbeitgeber eine große Gefahr, weil Beschlüsse der Kammer in die Welt hinausgehen können, ohne irgendwie entgegenstehende Ansichten der Minorität gezwungenermaßen zum Ausdruck zu bringen. Das liegt in der vom Entwurf vorgesehenen Organisation der Beschlu Bfassung der Kam$\mathrm{m}$ er $\mathrm{n}$, die nach $\$ 24$ Beschlüsse mit Stimmenmehrheit fassen und bei Stimmengleichheit den Vorsitzenden entscheiden läßt; Arbeitgeber und Arbeitnehmer müssen bei Beschlußfassung in gleicher Anzahl anwesend sein, Übersohießende der cinen oder anderen Interessengruppe scheiden aus. Es wird also in praxi der Verlauf einer BeschluBfassung meist so liegen, daß Arbeitgeber und Arbeitnehmer in kritischen Fragen sich nicht einigen werden, und daß damit die Fntscheidung in den Händen des Vorsitzenden liegen wird. Als Vorsitzenden bestellt der Entwurf einen Mann, der weder Arbeitgeber, noch Arbeitnehmer ist, in dubio also einen Beamten. Bei dem eben gekennzeichneten Verlauf der Verhandlung wird er, wem es ihm nicht gelingt, einen Beisitzer auf die entgegengesetzte Gruppe hinüberzuziehen, selbst den Ausschlag geben müssen, und damit ist der Grundgedanke der Parität illusorisch geworden, denn den Ausschlag gibt keine den Interessenten angehörende Person. Diese Schwierigkeit ist der Regierung auch zum Bewußtsein gekommen, denn am Schlusse des $\S 24$ heißt es : „Nehmen bei Erstattung eines Gutachtens gemäß $\$ 3$ Ziffer 2 (d. h. bei Gutachten zur Unterstützung von Staats- und Gemeindebehörden, die die Förderung gemeinsamer gewerblicher und wirtschaftlicher Interessen der Arbeit. geber und Arbeitnehmer zum Inhalt haben), oder 
bei Beratung eines Antrages gemäß $\$ 4$ (d. h. bei Anträgen an Behörden, an Vertretungen von Kom. munalverbänden und an die gesetzgebenden Körperschaften der Bundesstaaten und des Reiches) sämtliche Arbeitgeber einerseits und sämtliche Arbeitnehmer andererseits einen entgegengesetzten Standpunkt ein, so findet eine BeschluBfassung nicht statt." In allen anderen Fällen aber kann ein BeschluB der Kammer in der eben gekennzeichneten Weise zustande kommen, und die Ansicht der durch Stimmabgabe des Vorsitzenden überstimmten einen Seite kommt nicht zur Kenntnis der Allgemeinheit. Hierin liegt entschieden ein großer Fehler.

In diesem Zusammenhang ist der $\mathrm{W}$ a h $\mathrm{l}$ modus zuden Arbeitskammern einer kurzen Betrachtung zu unterziehen. Was berechtigt die die paritätischen Arbeitskammern ablehnenden Kritiker zu der Auffassung, daß die Gruppe der Arbeitnehmer sozialdemokratisch oder gewerkschaftlich beeinfluBt aus der Wahl hervorgehen wird? Nach $\$ 11$ und 12 des Entwurfs werden die Vertreter der Arbeitgeber von den Vorständen der Berufsgenossenschaften gewählt, die Vertreter der Arbeitnehmer 1. von den Mitgliedern der ständigen Arbeiterausschüsse nach $\$ 134 \mathrm{~h}$ der G. 0 . - als solche gelten die Vorstände der Fabrikkrankenkassen und die Knappschaftsältesten von Knappschaftsvereinen, sofern sie als ständige Arbeiterausschüsse bestellt werden, und die bereits vor dem 1./1. 1891 errichteten ständigen Arbeiteraussehüsse, deren Mitglieder in ihrer Mehrzahl von den Arbeitern aus ihrer Mitte gewählt werden, ferner solche Vertretungen, deren Mitglieder in ibrer Mehrzahl von den volljährigen Arbeitern der Fabrik aus ihrer Mitte in unmittelbarer und geheimer Wahl gewählt werden - und 2. aus den Vertretern der Arbeitnehmer, welche gemäß § 114 des Gewerbeunfallversicherungsgesetzes zur Beratung und Beschlußfassung usw. über Unfallverhütungsvorschriften bestellt sind.

Man wird nun nicht behaupten können, daß auf Grund dieser Wahlmöglichkeiten von vornherein zu sagen ist, daß eine geschlossene sozialdemokratische bzw. gewerkschaftliche Gruppierung der Arbeitnehmer in den Kammern gegeben ist. Das scheint schon daraus hervorzugehen, daß kein Paragraph des Entwurfes von der Sozialdemokratie und den Gewerkschaften so bekämpft und als unannehmbar bezeichnet wird, wie der über die Wahl der Arbeitnehmer. Immerhin ist das wahrscheinlich, daß es, wenn nicht gleich, so doch mit der Zeit aus folgenden Gründen zu dieser ausgesprochenen Gruppierung kommen wird. Der Entwurf hat offensichtlich versucht, die agitatorisch tätigen Organisationen der Arbeitnehmer möglichst auszuschalten - das wird ihm auch hauptsächlich von diesen zum Vorwurf gemacht -., das zeigt dje Kategorie der aus den Arbeiterbeiräten der Berufsgenossenschaften hervorgehenden Arbeitervertreter, die ans einer aus 7 Wahlhandlungen bestehenden Wahl hervorgeht, und die durchgängig von Sozialdemokratie und Gewerkschaften nicht als eine Vertretung angesehen wird, ,getragen vom Vertrauen ihrer Wähler", wie sie der Staatssekretär des Innern als notwendig für die Durchführung der gestellten Aufgaben verlangt.
Hier wird künftig also die Tätigkeit der Organisationen mit aller Macht einsetzen, um ihre Gesinnungsgenossen in diese Berufsgenossenschaftsbeiräte hineinzubringen, und ebenso wird das der Fall sein bei den oben unter 1. genannten Arbeiterausschüssen, wo dies nicht jetzt schon der Fall ist. Dann ist die Situation vorhanden, die die Arbeitgeberkörperschaften als nicht ersprießlich für ein gemeinsames Zusammenarbeiten ansehen.

So kommen also beide Teile, Arbeitgeber und Arbeitnehmer, zur Ablehnung der paritätischen Arbeitskammern, die ersteren, weil sie vorauszusehen glauben, daß die Gruppe der Arbeitnehmer sich künftighin nur aus gewerkschaftlich - bzw. sozialdemokratiseh beauftragten Elementen zusammensetzen wird, und die letzteren, weil nach ihrer Ansicht der organisierten Arbeiterschaft nicht die ihr zustehende Vertretung offiziell gesichert ist, and weil ferner nach ihrer Meinung nur in der reinen Arbeiterkammer ihre Ansichten unbeeinflußt von anderer Einwirkung zum Ausdruck kommen. Dazwischen steht die große Gruppe - ich möchte sagen - der Nichtinteressenten, die den Glauben an die Heilwirkung der Parität haben und jedenfalls als einen in Zukunft wirksamen Faktor ansehen.

Die Bestimmung der Wahl von Arbeitnehmern aus den ständigen Ausschüssen hat etwas Auffallendes, denn man darf wohl annehmen, daß man sich im Reichsamt des Innern gerade so wie in der Industrie bewußt ist, da $B$ ständige Arbeiterausschüsse auf Grund des $\$ 134$ h der G. $O$. noch so wenig existieren, daß man auf sie eigentlich kaum einen Wahlmodus aufbauen kann. Die Begründung des Entwurfes gibt hierzu die Erklärung in dem Satze : ,Wenn gleich zurzeit Arbeiterausschüsse noch nicht in der wünschenswerten Zahl bestehen, so fehlt es doch nicht an Anzeichen, daß ihre Errichtung in zunehmendem Maße als zweckmäßig erkannt wird." Dieser Gedanke wird also fernerhin energisch in allen Betrieben propagiert werden, und einzelne Parteien des Reichstages greifen jetzt schon der Regierung unter die Arme, wie der Antrag des Zentrums zur Novelle der Gewerbeordnung beweist, der eine obligatorische Einsetzung von Arbeiterausschüssen in allen Betrieben mit über 20 Arbeitern auf Grund freier und geheimer Wahl verlangt.

Nicht weniger wie die vorhergehenden Punkte ist auch die Frage umstritten: Sollen die A r be it skammern fachlich oder örtlich organisiert sein? Nachdem im Reichsamt des Innern als geeignetste Anlehnung für Schaffung von Arbeitskammern die Organisation der Berufsgenossenschaften angenommen worden war, war für dasselbe auch die fachliche Organisation gegeben. Auf die vielen Einwürfe, daß bei rein fachlicher Organisation die örtlichen Interessen der Arbeiterschaft zu den Arbeitgebern und zu den Gemeinden nicht genügend zum Ausdruck zu bringen sein würden, was unter allen Umständen wünschenswert sei, hat der Staatssekretär des Innern am $4 . / 3$, a. c. im Reichstag Gelegenheit genommen, zu ant,worten, und hat zugegegben, daß, diejenige Fühlung des Arbeiterstandes mit dem Arbeitgeberstand, mit den Verwaltungsbehörden, die erwünscht wäre, um manche Mißverständnisse $\mathrm{zu}$ beseitigen, in erster 
Linie hierbei nicht so gepflegt wird, wie es auch sein Wunsch wäre." Er hat aber nachzuweisen versucht, daß die rein territoriale Einteilung erheblich größere Schwierigkeiten und Nachteile für die sachgemäße Bearbeitung der den Kammern gestellten Aufgaben hätte, immerhin jedoch dabei in Aussicht gestellt, daß in Zukunft, wenn die beruflichen Arbeitskammern gut funktionieren, erwogen werden kann, ob aus ihnen nicht örtliche Ausschüsse als Unterorganisationen gebildet werden können. Dieses , in Zukunft" hat seine großen Bedenken, wenn man unter gutem Funktionieren der Kammern die Erreichung ihres Hauptzweckes versteht, der ja doch zweifellos die Herstellung eines guten Einvernehmens zwischen Arbeitgeber und Arbeitnehmer ist. Alle anderen im Entwurf angeführten Aufgaben sind ja wohl auch bemerkenswert, machen aber die Aufstellung eines derartigen umfangreichen Apparates kaum nötig; Gutachten, Anträge usw. kann sich die Regierung auf wesentlich einfachere Weise auch unter den jetzigen Verhältnissen verschaffen, Organisationen auf beiden Seiten sind hierfür genügend vorhanden. Diese Herstellung des Einvernehmens scheint doch wesentlich bedingt durch örtliche Organisation der Kammern, hjer wären in erster Linie die Momente gegeben, die bei entgegengesetzten Ansichten und bei ausbrechenden Streitigkeiten die Möglichkeit einer gegenseitigen Verständigung bieten : Kenntnis der lokalen Verhältnisse und Verständnis für die gegenseitigen Bedürfnisse und Möglichkeiten. Es würde also hier noch ein Weg zu suchen sein, der die fachliche Organisation wahrt und genügende Dezentralisation auf lokaler Grundlage gewährleistet.

Die Kosten d er Arbeits $\mathrm{kammern}$ werden durch den Entwurf den Berufsgenossenschaften, also den Arbeitgebern, auferlegt. Damit ist in bedenklicher Weise von der Parität abgewichen, und von beiden Interessentengruppen liegen dagegen Äußerungen vor. Auch hier hat Herr v. B ethm a n n - H o llwe g erklärt, daß das ein Vorschlag in Ermangelung eines besseren sei, und $\mathrm{da} B \mathrm{ihm}$ eine andere Regelung der Frage durchaus erwünscht sei.

Damit sind die Hauptpunkte des Entwurfes besprochen, es wäre noch zu erwähnen, daß das Gesetz nur Anwendung finden soll a uf Arbeitnehmer im Sinne der G. O. Tit. VII, d. h. nicht auf landw irtschaftliche Arbeiter, auch nicht auf Gehilfen im Handwerk, in Handelsgeschäften und in Apotheken. Betriebsbeamte, Werkmeister und Techniker sind nicht ausgenommen, jedoch ist für diese noch „ein besonderes Vorgehen in Aussicht genommen."

Wie aus dem Gesagten hervorgeht, befindet sich die Regierung vor der für sie wohl etwas überraschenden Tatsache, daß dje beiden Faktoren, zu deren Bestem gewissermaßen das Gesetz gemacht werden soll, den Grundgedanken des ganzen Entwurfs - den eines gemeinsamen Verhandlungskörpers - nahezu einstimmig ablehnen. Es tritt daher die Frage an sie heran, soll sie entgegen dieser entschieden ablehnenden Haltung der in Betracht kommenden Kreise und trotz dieser den Fn twurf aufrecht erhalten - denn mit der Frage der gemeinsamen Kammern steht und fällt der ganze Entwurf -? Das ist kaum anzunehmen, denn die Regierung, wie jedenfalls auch späterhin der Reichstag, wird sich der U̇berzeugung nicht verschließen können, daß die Voraussetzungen, die zu dem Entwurf in dieser Abfassung geführt haben, nicht zutreffen: Beide Interessentenvertretungen halten es unter den jetzigen Verhältnissen nicht für richtig, auf Grund des vorliegenden Entwurfes eine Förderung des wirtschaftlichen Friedens durch gemeinsame Beratung der Arbeitgeber und Arbeitnehmer in gemeinsamer Kammer zu erwarten. Dazu kommt, daß auch die Erfahrungen, die in Holland, Belgien und Italien mit den dort bereits bestehenden Arbeitskammern gemacht sind, keineswegs zur Durchführung des Gesetzes im Sinne des Entwurfes ermutigen können, denn wie dort, würden auch die deutschen Arbeitskammernn nur ein Scheindasein ohne nennenswerten Erfolg führon, weil es wie-dort keiner der Parteien einfallen würde, sich an den Verhandlungen zu betätigen, wenn es ihr in kritischen Fragen nicht paßt.

Der Gedanke, gegen solchen Widcrstand den Sitzungszwang einzuführen, wird abzulehnen sein aus der Auffassung heraus, auf der der ganze Entwurf gegründet ist. Alle Aufgaben der Kammer sollen gelöst werden und zum Ausdruck kommen auf Grund freier, nicht gezwungener Entschließungen nach bestem Wissen und Gewissen der Beisitzer. Der Wert solcher Entschließungen wird illusorisch, wenn diese auf andere als die eben gekennzeichnete Weise zustande kommen - ganz abgesehen davon, daß mit einem Sitzungszwang noch lange nicht die tatsächliche Beteiligung an der Verhandlung selbst und die Ausführung etwaiger Beschlüsse durch die Interessenten gegeben ist. -

Es könnte nun noch die Frage aufgeworfen werden, ob bei einer wesentlich engeren Begrenzung der Aufgaben der Arbeitskammern auf eine Zustimmung zur gemeinsamen Kammer zu rechnen wäre. Denn das ist kein Zweifel, es sind den Kammern in dem Entwurf eine große Anzahl Aufgaben zugewiesen, die in sich schon die Unwahrscheinlichkeit einer Verständigung tragen. Sieht man sich die Aufgaben der Kammern daraufhin an, so bleibt nicht viel; es könnten vielleicht in Betracht kommen Gutachten über einige wenige Punkte und Einrichtungen auf dem Gebiete der gewerblichen Wohlfahrtspflege, wo ja meist jetzt schon der einzelne Arbeitgeber seinen Arbeitern das Recht der Mitbetätigung zugesteht. Das sind aber für das allgemeine Wirtschaftsleben keineswegs so belangreiche Fragen, daB es der Mühe wert wäre, eine besondere Organisation dafür ins Leben zu rufen.

So bleibt meines Erachtens nur der Weg, entweder den Entwurf bis auf weiteres zurückzustellen oder auf die gemeinsame Kammer zu verzichten und in der reinen "Arbeiterkammer" den Arbeitnehmern eine gesetzliche Vertretung zu schaffen, in der sie ihre Bestrebungen und Wünsche vor der Öffentlichkeit zum Ausdruck bringen können, wozu die Arbeitgeber ja jetzt schon in den Handels-, Landwirtschafts- und Handwerkerkammern Gelegenheit haben. Und in diesem Gedanken finden sich beide Parteien in ihren Außerungen zum Entwurf auch zusammen, denn nicht nur dic Arbeitnehmer, sondern auch der größte Teil der Arbeitgeberschaft spricht sich dahin aus, daß, wenn überhaupt etwas geschaffen werden soll, die reine Arbeiterkammer das zurzeit Geeignetste ist. 